\title{
RÉSEAU D'ÉCOULEMENT A TRAVERS UN FORAGE INJECTÉ ET CONTRAINTES CORRESPONDANTES A LA FIN DE L'INJECTION
}

Dans un sol pulvérulent, nécessitant des injections d'imperméabilisation à base de silicates très fluides, le tracé du réseau d'écoulement peut présenter un certain intérêt pratique.

Dans un tel milieu non stratifié, les forages sont supposés suffisamment rapprochés pour transformer l'écoulement, qui est de révolution pour un seul forage, en un écoulement plan.

La détermination des lignes de courant et des équipotentielles est alors basée sur une représentation conforme appropriée ainsi que sur les propriétés bien connues des fonctions analytiques.

Au moyen de méthodes similaires, il est possible de trouver une solution élégante pour la répartition des contraintes dans le sol, au moment où le fluide d'injection ne peut plus pénétrer et développe à l'intérieur du forage une pression uniforme $« p »$.

\section{Transformation}

Soit $z=x+i y$ un plan passant par l'axe du forage. On appelle $w=u+i v$ un plan dans lequel les bords du forage peuvent être transformés en une ligne droite (fig. 1).

La représentation conforme :

$$
z=w \sqrt{w^{2}-1}-\operatorname{arcch} w+\frac{i \pi}{2}
$$

\footnotetext{
* Ingénieur docteur.

( $)$ H. Koner : "Dictionary of conformal representations》, Dover Publications, U.S.A.
}
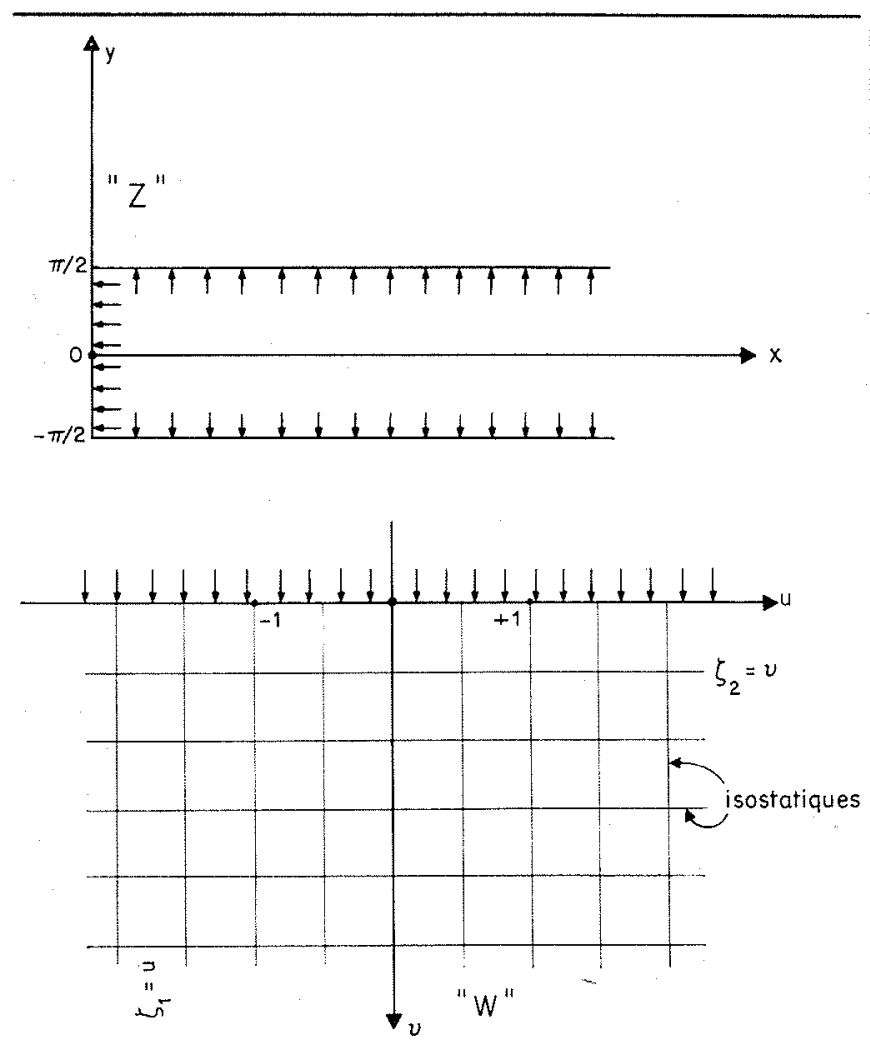

1/ Représentation conforme de l'espace $z$ en $w$ moyennant la transformation :

$z=w \sqrt{w^{2}-1}$ arech $w+i / 2$

Conformal representation of region $\mathrm{z}$ on $\mathrm{w}$, by the transformation :

$\mathrm{z}=\mathrm{w} \sqrt{\mathrm{w}^{2}-1} \operatorname{arccosh} \mathrm{w}+\mathrm{i} / 2$ 


\section{T. P. TASsios}

fait correspondre à tout point $z$, à l'extérieur du forage, un point $w$ du semi-espace infini, à condition que l'on tienne compte uniquement des valeurs positives de la fonction arc ch $w$.

C'est ainsi qu'aux deux familles de lignes droites perpendiculaires $\zeta(w)=w\left(u=\lambda_{i}\right.$ et $\left.\nu=\mu_{i}\right)$, du plan $w$, on fait correspondre le réseau d'écoulement cherché.

\section{Inversion numérique}

On forme les expressions analytiques de toutes les fonctions du $w=u+i \nu$ qui figurent dans l'expression de $z$.

On a :

$$
w^{2}-1=\mathrm{R}_{1}+i \mathrm{R}_{2}
$$

où

$$
\begin{gathered}
\mathbf{R}_{1}=u^{2}-v^{2}-1 \quad \text { et } \mathrm{R}_{2}=2 u v \\
\sqrt{w^{2}-1}=\rho \cos \frac{\theta}{2}+i \rho \sin \frac{\theta}{2} \\
\rho=\sqrt[3]{\mathbf{R}_{1}^{2}+\mathbf{R}_{2}^{2}} \quad \text { et } \theta=\operatorname{arctg} \frac{\mathrm{R}_{2}}{\mathrm{R}_{1}}
\end{gathered}
$$

$w \cdot \sqrt{w^{2}-1}=\left(u p \cos \frac{\theta}{2}-v \rho \sin \frac{\theta}{2}\right)+$

$$
+i\left(v \rho \cos \frac{\theta}{2}+u p \sin \frac{\theta}{2}\right)
$$

$\operatorname{arcch} w=\operatorname{arcch} \mathrm{S}+i \operatorname{arcch} \frac{u}{\mathrm{~S}}$

où $\quad \mathrm{S}=\frac{1}{2}(k+q) \quad k=\sqrt{(1+u)^{2}+v^{2}}$

$$
\text { et } q=\sqrt{(1-u)^{2}+v^{2}}
$$

La fonction $z$ prend alors la forme suivante :

$$
\begin{aligned}
& z=x+i y= {\left[u \rho \cos \frac{\theta}{2}-v \rho \sin \frac{\theta}{2}-\operatorname{arcch} \mathrm{S}\right]+} \\
&+i\left[v \rho \cos \frac{\theta}{2}+u \rho \sin \frac{\theta}{2}-\operatorname{arcch} \frac{u}{\mathrm{~S}}+\frac{\pi}{2}\right]
\end{aligned}
$$

Dans le tableau qui suit, nous avons calculé les valeurs des $x, y$ correspondant aux lignes $u=\lambda_{i}$ et $\nu=\mu_{i}$. La figure 2 représente le réseau d'écoulement cherché, dans un plan axial du forage.

\section{Contraintes du sol dans le cas d'une injection « bouchée "}

D'après P. Nemenyi (Strömlinien und Hauptspannungstrajektorien, Zeitschrift für Angew., Math. and Mech., Okt. 1933), ou plus récemment P. Théocaris (Direct détermination of stresses in plane elasticity problems based on the properties of isostatics, J. Appl. Mech., June 1959), en appliquant la même méthode, on pourrait calculer les contraintes sur le squelette solide, au moment où ses pores sont bouchés et une pression uniforme $p$ agit le long des bords du forage. Dans ce cas, on aurait, sur le plan $z$ :

$$
\begin{aligned}
\sigma_{x, y}= & \mathrm{R} \\
& \pm\left[a+b x+c y+d\left(x^{2}+y^{2}\right)\right] \cdot \mathrm{R}\left[\left(\frac{\partial \zeta}{\partial z}\right)^{2}\right]
\end{aligned}
$$

$\tau_{x, y}=2\left[{ }^{-} a+b x+c y+d\left(x^{2}+y^{2}\right)\right] . \mathrm{J}\left[\left(\frac{\partial \zeta}{\partial z}\right)^{2}\right]$

où $\quad \mathrm{R}, \mathrm{J}=$ partie réelle et partie imaginaire de la fonction correspondante;

$\zeta(w)=$ les isostatiques (ou lignes du réseau d'écoulement) sur le plan $w$;

$a, b, c, d=$ constantes à déterminer.

Dans notre cas nous avons $\zeta(w)=w$ et :

$$
\frac{\partial \zeta}{\partial z}=\frac{\partial \zeta}{\partial w} \cdot \frac{\partial w}{\partial z}=\frac{1}{2 \sqrt{w^{2}-1}}, \quad \text { ou }\left(\frac{\partial \zeta}{\partial z}\right)^{2}=\frac{1}{4\left(w^{2}-1\right)}
$$

On aura également :

$$
\begin{array}{r}
\int(2 d z+b+i c) \cdot\left(\frac{\partial \zeta}{\partial z}\right)^{2} d z=\frac{d}{2}\left[\begin{array}{c}
w^{2}-(\operatorname{arcch} w)^{2}+ \\
+i \pi \log \\
\left(w+\sqrt{\left.w^{2}-1\right)}\right.
\end{array}\right] \\
+\frac{b+i c}{2} \log \left(w+\sqrt{\left.w^{2}-1\right)}\right.
\end{array}
$$

On obtient ainsi l'expression suivante pour les contraintes $\sigma_{x}, \sigma_{y}$ et $\tau_{x y}$ :

$$
\begin{aligned}
\sigma_{x, y}= & \left.-\left\{\begin{array}{r}
\frac{d}{2}\left[\left(\mathrm{R}_{1}+1\right)-\mathrm{M} \cos 2 \gamma-\pi \varphi\right. \\
+\frac{1}{2}[b \log \mathrm{P}-c \varphi
\end{array}\right]\right\} \\
\pm & \frac{1}{2}\left[a+b x+c y+d\left(x^{2}+y^{2}\right)\right] \cdot \frac{\mathrm{R}_{1}}{\rho^{4}} \\
\tau_{x, y}= & -\frac{1}{2}\left[a+b x+c y+d\left(x^{2}+y^{2}\right)\right] \cdot \frac{\mathrm{R}_{2}}{\rho^{4}}
\end{aligned}
$$

où :

$$
\begin{aligned}
\Upsilon & =\operatorname{arctg} \frac{\operatorname{arcch}(u / S)}{\operatorname{arcch} \mathrm{S}} \\
\mathrm{M} & =[\operatorname{arcch} \mathrm{S}]^{2}+\left[\operatorname{arcch} \frac{u}{\mathrm{~S}}\right]^{2} \\
\mathrm{P} & =\sqrt{\left(u+\rho \cos \frac{\theta}{2}\right)^{2}+\left(v+\rho \sin \frac{\theta}{2}\right)^{2}} \\
\varphi & =\operatorname{arctg} \frac{v+\rho \sin \theta / 2}{u+\rho \cos \theta / 2}
\end{aligned}
$$

Après avoir déterminé les constantes $a, b, c, d$, par les conditions aux limites (par exemple, pour $x=0, \sigma_{x}=-p$ et $\tau_{x y}=0$, etc.), on effectue le calcul numérique des contraintes. Généralement, l'application des fonctions analytiques dans la théorie de l'élasticité conduit à des expressions de contraintes qui laissent les valeurs parasitaires très importantes sur les bords.

A l'aide d'une des méthodes connues on procède ensuite au «nettoyage» approximatif de ces bords.

\section{Vérification expérimentale du réseau des isostatiques}

Les courbes théoriques du réseau d'écoulement (fig. 2), ou courbes isostatiques dans le cas de sollicitation des parois bouchées, ont été vérifiées expérimentalement à l'aide d'un modèle électrique sur papier recouvert de graphite (fig. 3 ). 

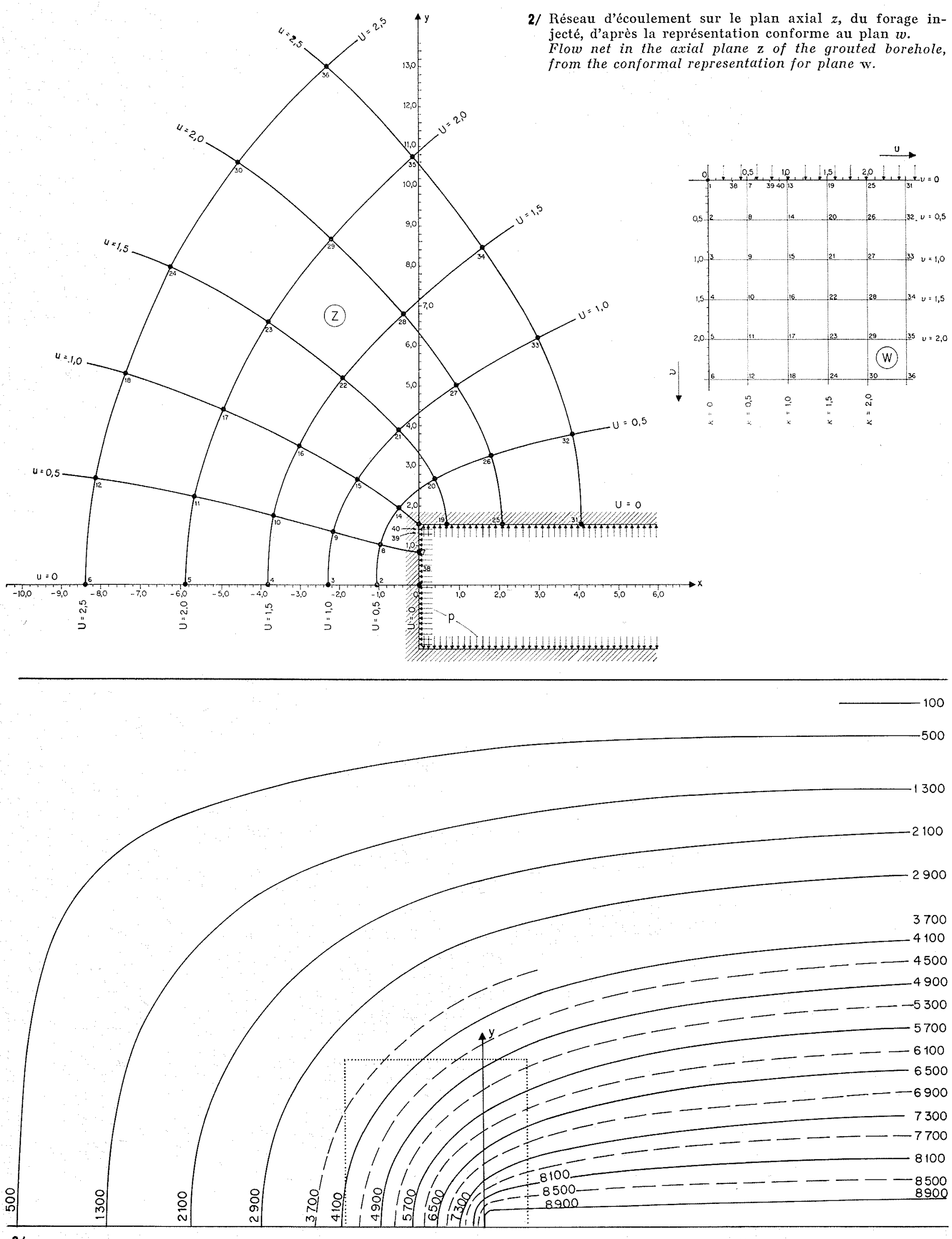


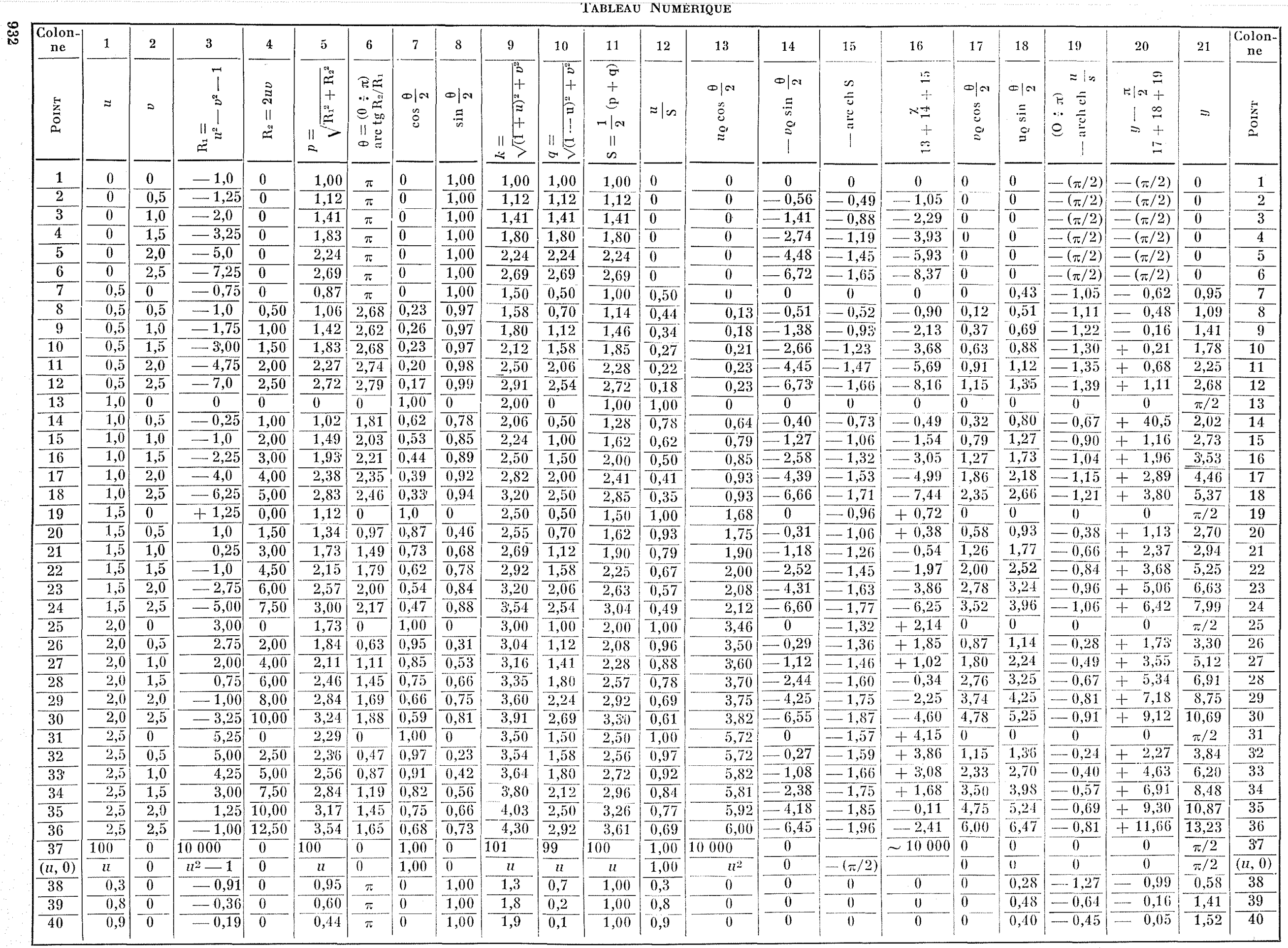




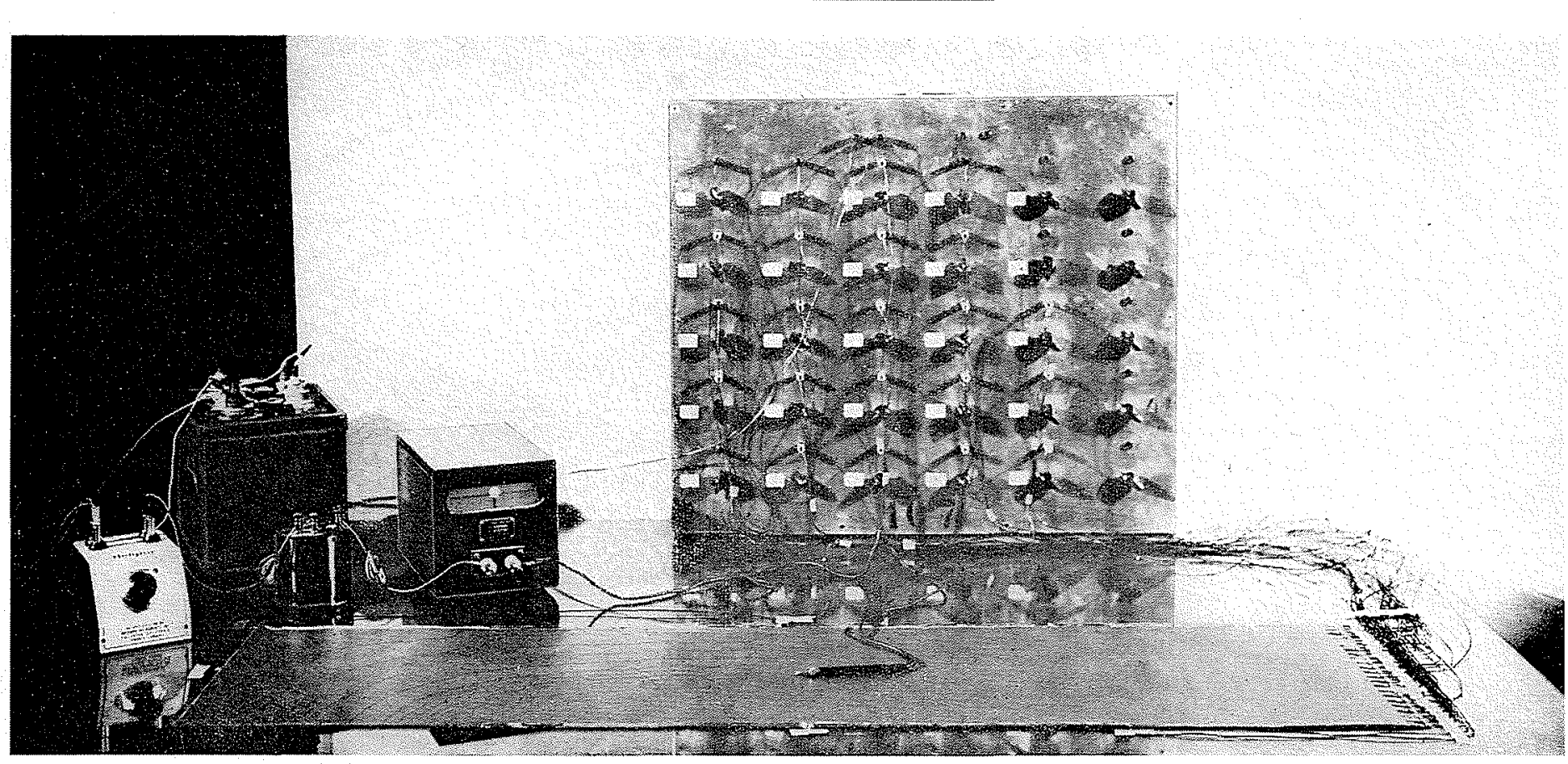

4/

La photo figure 4 du modèle avec les tracés des équipotentielles électriques, constitue une vérification de la figure théorique (fig. 2), au moins pour les parties au voisinage de la pointe.

Les mesures correspondantes ont été réalisées dans le Laboratoire de Résistance des Matériaux de I'Université Technique d'Athènes.

L'auteur désire remercier le professeur P. Théocaris pour son aide et l'intérêt qu'il a porté à cette étude. 